\title{
The Basics of Ultrasonography
}

\section{Rashid $S Q^{l}$}

\begin{abstract}
Ultrasound is sound whose frequency is above the human hearing range. It is nowadays widely used for the evaluation of a patient's internal organs. Ultrasound waves are transmitted into the human body by an instrument called the transducer. Inside the body the sound waves are reflected and scattered differently by the different tissues and organs. The reflected sound waves are used by the computer to form an image of the internal structures and tissues. Use of ultrasound is safe with negligible bio-effects.
\end{abstract}

\section{INTRODUCTION}

Ultrasonography is one of the new tools that we have acquired for diagnostic purpose. It is the brainchild of Dr. Ian Donald who was a gynecologist by profession. His invention has brought newer dimensions to the diagnostic field of not only obstetrics and gynecology but also to the other fields of medicine like internal medicine, surgery, ophthalmology, orthopedics, cardiology etc. and more and more fields are coming into its folds for not only diagnosing diseases but also for therapeutic purpose.

It is a very popular modality with both the physicians as well as the patients. Reason being it is cheap, easily available, non-invasive, and with no radiation hazard. Also because some of the images that it displays on the screen are quite easily understood by the patients even. So that when a pregnant lady views her baby still inside her womb for the first time her joy and her husband's too is quite obvious. The thrill of seeing their baby moving, its heart beating, gives them so much of happiness. A top film star's husband after seeing their baby inside his wife's womb was so excited that he wanted to buy one such machine and keep it at his house!

Then the sight of a moving worm swimming in the bile inside the gall bladder is amazing even for the doctor performing the scan.

\section{ULTRASONOGRAPHY:}

\section{Physics of Ultrasound}

The basic principle of all ultrasound imaging is that, the ultrasound waves are send into the body, it is then reflected

1. Dr. Sabrina Q Rashid, Assistant Professor, department of radiology, University of Science \& Technology Chittagong., Dhaka.

Tel: 9116940 (C), 8825365 (R). back from the interfaces of the organs and tissues. The reflected waves are then detected and displayed as an image on the monitor.

To produce, detect and process ultrasound data, numerous variables, many under direct user control, must be managed. To do this the user must understand the methods used to generate ultrasound data and the theory and operation of the instruments that detect, display and store the acoustic information generated in clinical examinations. 1

\section{Basic Acoustics}

Sound is the result of mechanical energy traveling through matter as a wave, producing alternating compression and rarefaction. In the use of ultrasound to produce image of body tissues and organs brief bursts of energy are transmitted into the body where it propagates through the tissue. In the body, propagation velocity of sound is determined by the physical properties of tissue. The velocity of sound in tissues is increased by increase in stiffness and reduced by increase in density. The propagation velocity of sound is assumed to be $1540 \mathrm{~m} / \mathrm{sec}$. This value is the average of measurements obtained from normal soft tissues.2 Propagation velocity of sound is $4080 \mathrm{~m} / \mathrm{sec}$ in bone and $330 \mathrm{~m} / \mathrm{sec}$ in air. Propagation velocity is important in clinical ultrasound as it is used to determine the distance of the interface or the tissue from the transducer.

To produce an echo, a reflecting interface must be present. Sound passing through a totally homogenous medium, like water, encounters no interface to reflect sound, and the medium appears anechoic or cystic. When sound passes from one tissue to another, some of the incident sound energy is reflected. 1 The way ultrasound is reflected when it strikes an acoustic interface is determined by the size and surface features of the interface. If the interface is large and relatively smooth, it reflects sound much as a mirror reflects light. They are called specular reflectors, eg. Diaphragm, and walls of a full urinary bladder. Most echoes in the body do not arise from specular reflectors but come from smaller interfaces within solid organs. Interfaces that are either smaller than the wavelength or not smooth are nonspecular .(Figure-1) The echoes from these nonspecular interfaces are scattered in all directions. They are also called diffuse reflectors and produce the echoes that form the echo patterns seen in different solid organs and tissues eg. liver parenchyma, red blood cells, and other materials representing the tissue characteristics of an organ. ${ }^{3}$ 

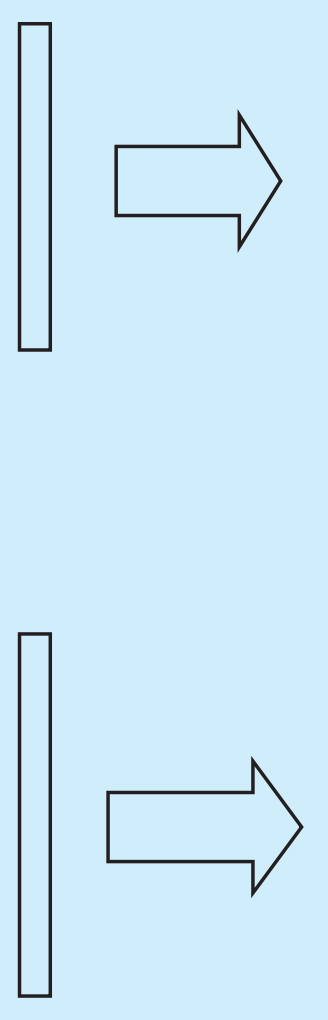

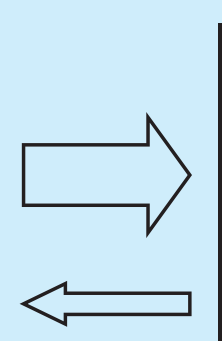

Specular reflection

\section{Large reflector}

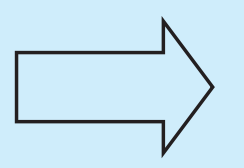

Small Reflector

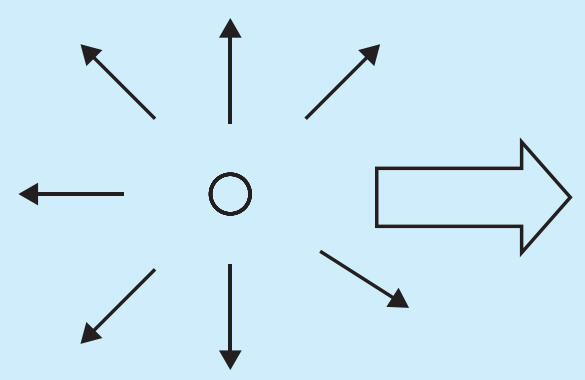

Scattering

Figure-1: Two distinct patterns of reflection give rise to the echoes that make up an ultrasound image - specular reflection and scattering.

\section{Ultrasound Pulse}
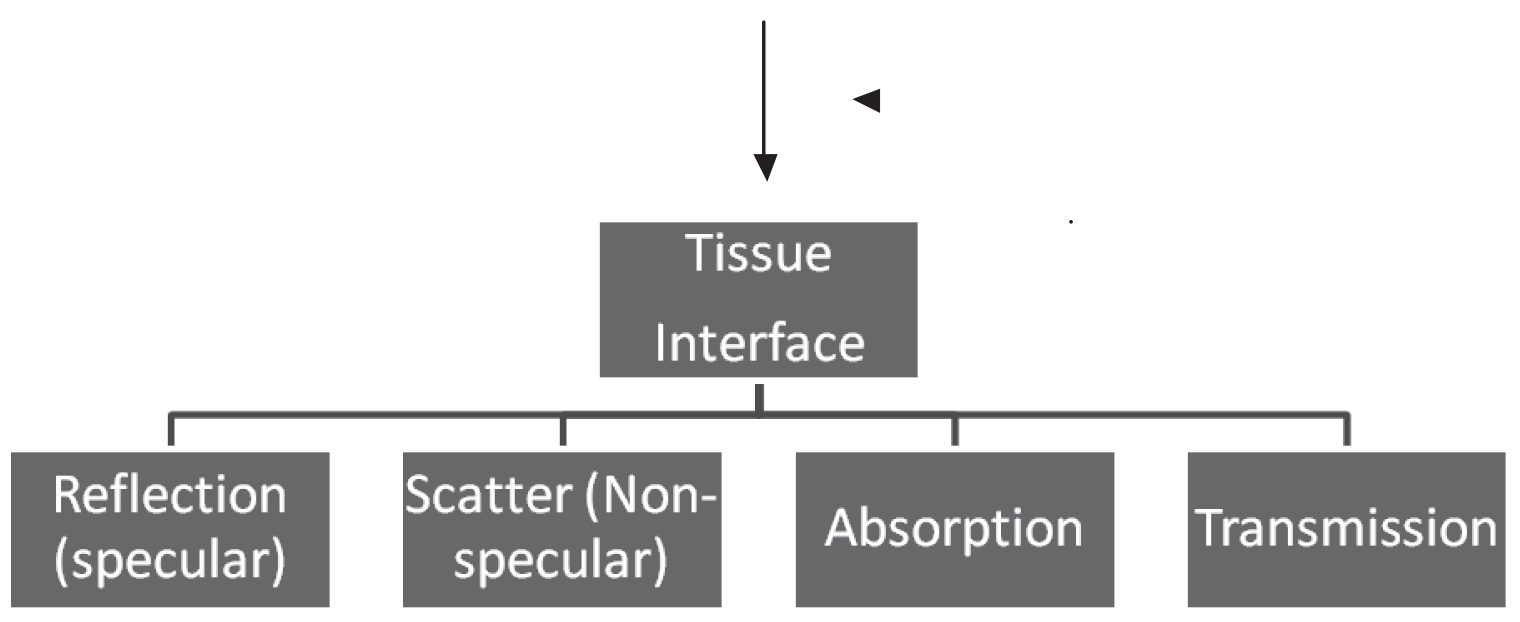

Figure- 2 : Illustration of phenomena which result when an ultrasound pulse encounters a tissue interface. 
Resolution is the minimum reflector separation required to produce separate reflections in a pulse-echo system. Attenuation is the decrease in energy as a wave travels through a medium. Attenuation is caused by absorption, reflection, beam divergence and scattering. In bone attenuation is greater but in water it is very low. 3 As sound passes through tissue it loses energy and pressure waves decrease in amplitude as they travel further from their source. Attenuation is therefore the result of the combined effects of absorption, scattering, and reflection. (Figure-2) High frequencies are attenuated more rapidly than lower frequencies, and transducer frequency is a major determinant of the useful depth from which information can be obtained with ultrasound. ${ }^{1}$

High frequency transducers are used to generate the ultrasonic energy. The major component of an ultrasound transducer is the piezoelectric element. Piezoelectric materials are capable of converting one form of energy into another. A voltage supplied to a piezoelectric element initiates vibrations. Its another function is to receive echoes that return from the object being studied. It than converts this mechanical energy into an electrical voltage, which forms a visual image of the studied structure. Transducers come in many different frequencies- typically 2.5, 3.5, 5, 7 and $10 \mathrm{MHz}$. Increasing the frequency improves resolution but decreases penetration. Decreasing the frequency increases the penetration but diminishes resolution. ${ }^{4}$

Transducers have an effect on image. Therefore transducers are chosen according to the structure being examined and the size of patient. Pediatric patients can be examined at 5 to 7.5 MHz. Lower frequencies (eg. $2.5 \mathrm{MHz}$ ) permit greater penetration and may be needed to scan larger patients. ${ }^{4}$

Doppler is used to detect blood flow through vessels. The Doppler technique detects not only the presence of blood flow but also the direction of flow by measuring the difference in the frequency of the reflected sound compared with the transmitted sound. ${ }^{3}$

As a general rule, medical diagnostic ultrasound energy will not travel through air. This is why acoustic couplants (water, oil or gel) are needed. Acoustic couplants are used to provide a good sound path between the transducer and the skin.3

\section{Bioeffects}

Bioeffects or biological effects are the effects of ultrasound on tissue. The categories of bioeffects are

1. Heat

2. Cavitation

\section{Others}

Heat is the effect created by the motion of the vibrating molecules. Any heating is negligible when pulse ultrasound is used for diagnostic purposes. Cavitation results in the production of gas bubbles. It can result in damage of cell walls. 'Others' include various minor mechanical effects that are not related to heat or cavitation.3

\section{Instrumentation}

Types of ultrasound display

A-mode or amplitude mode is the most basic form of diagnostic ultrasound. Its use is almost obsolete.

In B-mode or brightness mode a stronger (high amplitude) echo will display a brighter dot than a weaker (low amplitude) echo. The depth of the reflector is displayed by the location of the dot. 5 An image of the organ or tissue is formed in gray-scale as different dots of different intensities appear on the monitor.

In M-mode or motion mode, a series of B-mode dots is displayed on a moving time base graphing the motion of mobile structures. M-mode is currently used in conjunction with real-time imaging in adult, pediatric and fetal echocardiography. ${ }^{5}$

\section{Types of Transducers}

Mechanical Transducers. The transducer crystal is physically moved. Most of these provide a sector image with a fixed focus. It is not now commonly used in modern equipment.

Electronically steered systems. In this type of transducer, multiple piezoelectric elements are used. A separate electrical supply is provided for each element. Focusing is controlled electronically. The images are displayed in a sector, linear or curved-linear format. ${ }^{5}$ (Figure-4)

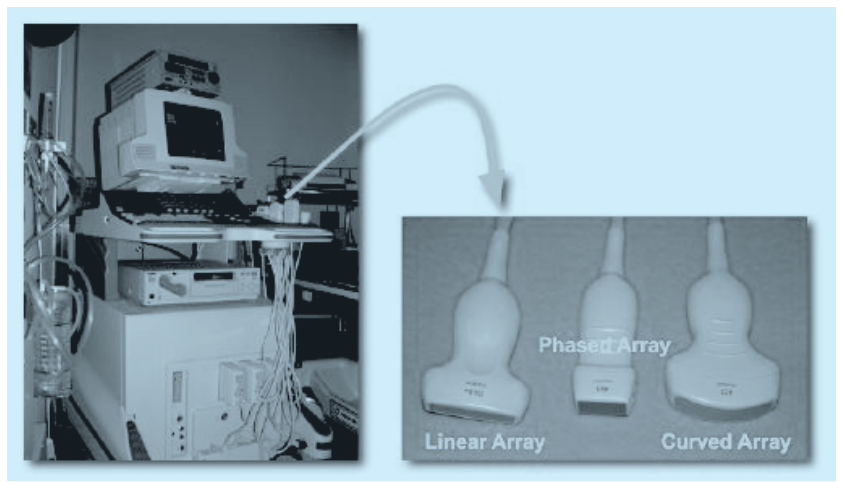

Figure-4 : Photos of a sonography system and typical transducers:

\section{Specialized Ultrasound Systems}

Small Parts Scanners use probes capable of high- resolution, for example 7.5 to $10 \mathrm{MHz}$ transducers. They are designed for visualizing the fine details of superficial structures, usually at a depth of less than $4 \mathrm{~cm}$ from the skin surface (eg. Thyroid, carotid arteries, testes, breast or structures in an infant).

Endo-ultrasound Systems (Trans-vaginal and trans-rectal probes). The transducer is placed on the end of a rod. This is then inserted into the vagina or rectum.

Endo-luminal or trans-luminal transducers. Even smaller transducers on the end of catheters can be introduced into vessels, the billiary duct or the ureters.

Operative Systems. Probes are modified to be used in sterile 
fashion in the operating room. Special high-frequency ultrasound probes are used for this purpose. 5

Doppler ultrasound instruments are optimized to display flow information. The simplest Doppler devices use continuous wave rather than pulsed wave ultrasound, using two transducers that transmit and receive ultrasound continuously (continuous wave or CW Doppler). [1] The most common form of Doppler ultrasound to be used is color flow Doppler imaging.6 Signal phase provides information about the presence and direction of motion, and changes in echo signal frequency relate to the velocity of the target. Flow within the vessel is observed at all points, and stenotic jets and focal areas of turbulence are displayed.1

\section{CONCLUSIONS}

In conclusion, ultrasound is one of the latest technologies given to the doctors and patients by modern science. But in the hands of an ill trained or minimally trained physician it is a bane rather than a boon for both the doctor and the patient. Because the misdiagnosis of important and emergency or life threatening diseases can greatly harm the patient e.g. mis-diagnosing an ectopic pregnancy, a major fetal anomaly or masses in the organs. The bio-effects of ultrasound is essential to understand but it is minimum in the modern machines compared to the hazards of radiation in other diagnostic modalities.

It is a versatile tool in the hands of the physician and can give him/her a wealth of information in the management of the patient in the best possible manner. It can be used without hesitation for the benefit of the patient. Ultrasound is now also used for therapeutic purpose as for crushing gall bladder and renal stones.

Ultrasound is a very practical and personal skill, which take a considerable amount of practice to perfect.

\section{REFERENCES}

1. Merritt CRB. Physics of Ultrasound. In: Rumack CM, Wilson SR, Charboneau JW (eds.), Diagnostic Ultrasound, 2th Ed. St. Louis. Mosby, Inc. 1998, p 3-33.

2. Chivers RC, Parry RJ. Ultrasonic velocity and attenuation in mammalian tissues. J Acoust Soc Am 1978; 63:940-53.

3. Pinkney MN. Physics. In: Curry RA, Tempkin BB (eds.), Sonography, 2th Ed. St. Louis. Saunders, Elsevier. 2004, p 17-22.

4. Sanders RC. Basics. In: Sanders RC, Miner NS (eds.), Clinical Sonography, 3rd Ed. Philadelphia. Lippincott. 1998, p 2-7.

5. Saylor MM, Sanders RC. Instrumentation. In: Sanders RC, Miner NS (eds.), Clinical Sonography, 3rd Ed. Philadelphia. Lippincott. 1998, p 8-21.

6. Merritt CRB. Doppler color flow imaging. J Clin Ultrasound 1987;15:591- 7. 